\title{
RA autoantibodies as predictors of rheumatoid arthritis in non-cystic fibrosis bronchiectasis patients
}

To the Editor:

The mechanisms underlying the strong association between rheumatoid arthritis (RA) and bronchiectasis were recently reviewed [1]. A literature review highlighted 289 reports of bronchiectasis associated with RA, with the respiratory symptoms preceding joint symptoms in $90 \%$ of the reports [2], strongly suggesting that the processes in bronchiectasis predispose to RA.

Rheumatoid factor (RF) and anti-cyclic citrullinated antibody (anti-CCP) are integral in the initiation of RA. A Danish study $(n=9712)$ observed that the baseline IgM RF was predictive of a significant, six-fold increased risk of RA development if the RF was two- to four-fold above the normal range [3]. Likewise, a strongly positive anti-CCP test significantly increases the risk of RA (OR 25) [4].

Two studies have investigated RF in bronchiectasis $[5,6]$ with no studies to date investigating anti-CCP. In our BRAC RA (Bronchiectasis, Asthma, Control, Rheumatoid Arthritis) study, a prospective, multicentre, case-control, observational study was conducted to determine the relationship between bronchiectasis and RF and anti-CCP. Recruitment was completed over 12 months using identical methodology and reviewed by the same researcher (E. Perry) with full ethical approval (Integrated Research Application System approval number 12324; Health Research Authority, London, UK). All recruited bronchiectasis patients were under respiratory specialist care, and had high-resolution computed tomography (HRCT) evidence of bronchiectasis and a history of two or more respiratory infections per year. Key exclusion criteria included inflammatory arthritis, tuberculosis or other forms of lung disease. Cystic fibrosis was excluded using genotyping and sweat testing following British Thoracic Society guidelines. Asthma patients were identified from a database where the diagnosis had been confirmed by expert review. Healthy controls were matched with the asthma patients, where possible, for smoking history, age and sex. Anti-CCP measurement was undertaken by ELISA assays including the EliA CCP second-generation ELISA kit (Phadia, Uppsala, Sweden) and IgM RF was quantified using a Modular P analyser (Hitachi, Maidenhead, UK) with cut-off levels as per the manufacturer's instructions. As per the 2010 American College of Rheumatology (ACR)/ European League Against Rheumatism (EULAR) criteria for RA [7], a negative result was defined as within the normal range, a "weakly positive" result as greater than the upper range of normal but below the level of three times the upper range of normal, and a "strongly positive" result as greater than three-fold higher than the upper range of normal. All individuals underwent analysis for RF and anti-CCP. Individuals who developed RA according to the 2010 ACR/EULAR criteria over 12 months were noted [7].

In total 122 bronchiectasis patients, 87 asthma patients and 78 controls were studied. RF positivity was significantly more prevalent in bronchiectasis compared with controls (31 (25.4\%) out of 122 versus eight $(10.3 \%)$ out of $78, \mathrm{p}=0.01)$ but not significantly different when compared with the asthma patients (14 $(16.1 \%)$ out of $87, p=0.13)$. Of those $31 \mathrm{RF}$-positive bronchiectasis patients, four (13\%) were strongly antiCCP positive, versus none of the $91 \mathrm{RF}-$ negative bronchiectasis patients $(\mathrm{p}=0.0036)$. A strongly positive anti-CCP occurred significantly more frequently in bronchiectasis patients (four (3.3\%) out of 122 versus none out of 164 of the asthma and control cases combined, $p=0.03$ by Fisher's exact probability test). Of these four bronchiectasis patients strongly positive for anti-CCP, two subsequently developed RA within 12 months. The demographic details of the bronchiectasis patients, asthma patients and controls are summarised in figure 1 a.

Undoubtedly, a positive RF and anti-CCP in non-RA individuals greatly increases the risk of RA development $[3,4]$. Therefore, our findings of a significantly more prevalent positive RF and anti-CCP in the bronchiectasis patients may explain the reported relationship between bronchiectasis and RA 


\begin{tabular}{lccccc}
\hline a) Group & $\begin{array}{c}\text { Age years } \\
\text { median (IQR) }\end{array}$ & $\begin{array}{c}\text { Females } \\
\mathbf{n}(\%)\end{array}$ & $\begin{array}{c}\text { Current/ } \\
\text { ex-smokers } \mathbf{n}(\%)\end{array}$ & $\begin{array}{c}\text { Disease duration } \\
\text { median (IQR) }\end{array}$ & $\begin{array}{c}\text { FVC \% pred } \\
\text { median (IQR) }\end{array}$ \\
\hline $\begin{array}{l}\text { BR } \\
\text { (n=122) }\end{array}$ & $66(13)$ & $80(65)$ & $48(39)$ & $10.98(25)$ & $85(30)$ \\
$\begin{array}{l}\text { Asthma } \\
\text { (n=97) } \\
\begin{array}{l}\text { Control } \\
\text { (n=78) }\end{array}\end{array}$ & $50(27)$ & $69(79)$ & $37(43)$ & $30.12(22)$ & $86(32)$ \\
\hline
\end{tabular}
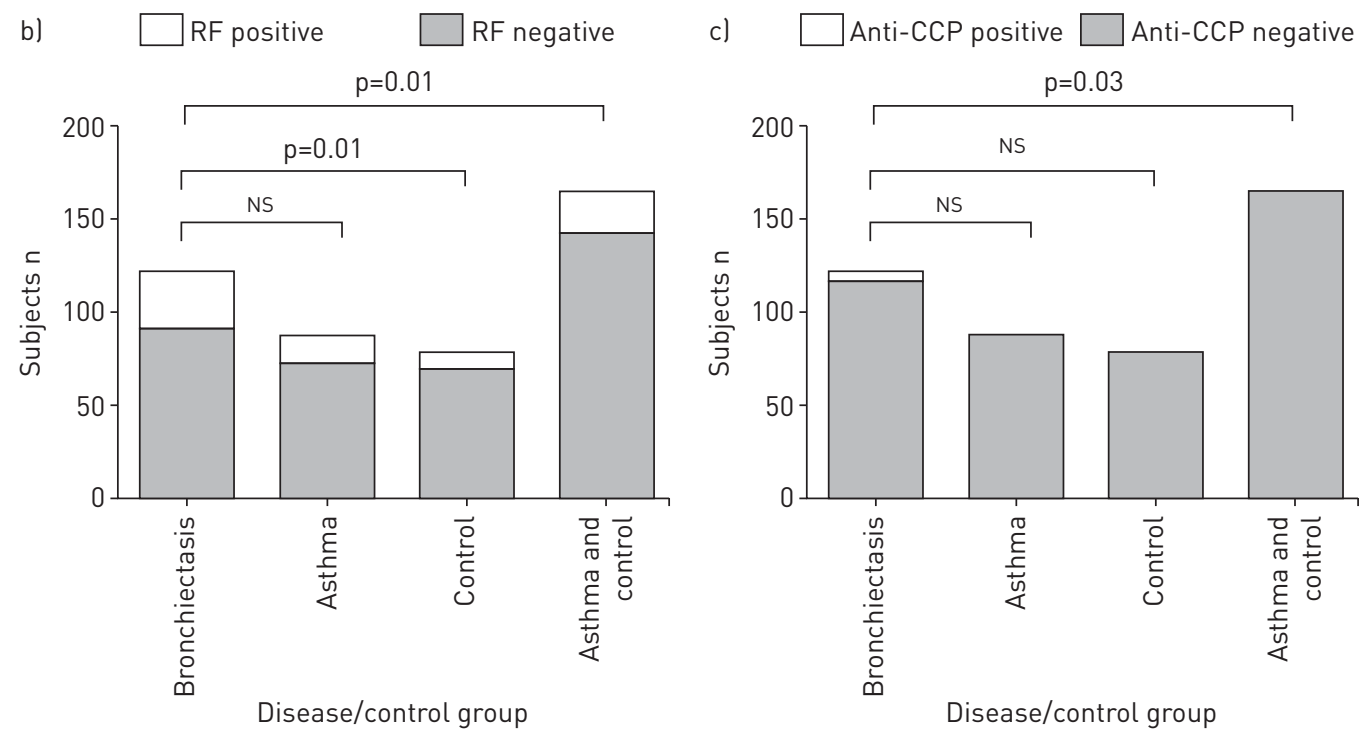

FIGURE 1 Demographic and functional test parameters data and prevalence of rheumatoid factor (RF) and anti-cyclic citrullinated antibody (anti-CCP) antibodies in a non-cystic fibrosis population of bronchiectasis (BR) patients. a) In terms of RF and/or anti-CCP, there was no difference in age between the patients who were found to be RF and/or antiCCP positive (median 62.72 years, interquartile range (IQR) 19.97 years) and those who were RF and/or anti-CCP negative (median 61.90 years, IQR 19.69 years). Therefore differences in RF/anti-CCP between the groups would not appear to be attributable to age. b) Presence of RF autoantibodies and c) a strongly positive anti-CCP occurred significantly more frequently in BR patients four (3.3\%) out of 122 versus none out of 165 of the asthma and control cases combined ( $p=0.03$ by Fisher's exact probability test). A two-sided $p$-value of less than $5 \%$ was considered significant. FVC: forced vital capacity; NS: nonsignificant.

development [2]. To date, smoking has been regarded as the strongest risk factor for the development of a positive RF test in the general population, with a Finnish study $(n=7124)$ observing RF positivity to occur twice as often in smokers than nonsmokers (a positive RF was observed in only $2.8 \%$ of the nonsmokers) [8]. The majority of the bronchiectasis patients in our study had never smoked (61\%) yet $18 \%$ of these patients were RF positive, suggesting that bronchiectasis is associated with RF independently of smoking. Furthermore, only $4.9 \%$ of Danish current smokers derived from the general population have a positive RF test [3]; our data therefore suggest that bronchiectasis has a closer association with RF than smoking in individuals without RA. Our data do not suggest that bronchiectasis is the strongest environmental association with RF positivity. One prior study demonstrated RF positivity in $42 \%$ of chronic obstructive pulmonary disease (COPD) patients [9], suggesting an interaction between cigarette smoking, COPD and RF production; however, none of these individuals were anti-CCP positive, unlike the bronchiectasis patients reported here.

Hilton and Doyle [5] observed a positive RF test in $52 \%$ of their 52 bronchiectasis patients, and HorAN et al. [6] in $29 \%$ of their 24 bronchiectasis patients. These prior studies, in contrast to ours, included patients with connective tissue diseases, which are associated with RF positivity [10]. Secondly, both prior studies included RA patients. Finally, the diagnostic threshold for bronchiectasis has changed dramatically with the advent of HRCT scanning, which unquestionably leads to an earlier diagnosis. The clinical characteristics of the cohorts of Hilton and Doyle [5] and Horan et al. [6] support the supposition of a more severe bronchiectasis disease burden with relatively severe airflow obstruction and very high rates of Gram-negative pathogens observed in $95 \%$ and $52 \%$ of cases, respectively. 
Both the asthma and bronchiectasis patients had a RF positivity rate higher than the control population. This is not altogether surprising as the presence of lymphoid follicles is noted in both asthma and bronchiectasis patients $[11,12]$. Lung lymphoid tissue has been observed to be associated with local RF production [13]. Our asthma patients were younger than the bronchiectasis patients: if the asthma and bronchiectasis groups were more closely age matched, we may have had greater confidence in concluding both these separate groups truly had similarly raised levels of RF (fig. 1b). An alternative explanation for our findings of raised RF in asthma reflects a limitation of our study: we did not limit recruitment to those asthma patients with a normal HRCT. The rates of HRCT scanning in asthma were very low in general, and patients with evidence of bronchiectasis and a history of asthma were excluded. It is conceivable, however, that patients who had developed bronchiectasis as a complication of their asthma (but symptoms did not prompt a clinical need for computed tomography) were included in our study group as asthma alone. This may explain why we failed to demonstrate a significant difference between the bronchiectasis and asthma patients in terms of a weakly positive RF. A blood donor study investigating RA development studied 2138 controls; $12(0.6 \%)$ were anti-CCP positive [14]. In contrast, in our study, a positive anti-CCP was almost six-fold more prevalent $(3.3 \%)$ in our bronchiectasis patients and was exclusively associated with RF positivity. This is important as a combination of both RF and anti-CCP was associated with a $100 \%$ conversion rate to RA in the aforementioned blood donor study over 5 years, as compared with a conversion rate of $1.5 \%$ for blood donors with only a positive IgM RF over the same period [14]. The expected rate of RA development in a Dutch general population was one in 1000 over 5 years [15], whereas we observed RA development in bronchiectasis of two out of 122 over 1 year.

BRAC RA represents the largest study of RF and the only study of anti-CCP in a well-characterised cohort of bronchiectasis patients. The key findings from this study are: 1) RF positivity is significantly more prevalent in bronchiectasis patients compared with healthy controls; 2) there is a significant association between RF positivity and anti-CCP positivity in bronchiectasis patients; 3) strongly positive RF and anti-CCP antibodies that are known to confer a high risk of RA development were significantly more prevalent in bronchiectasis patients compared with the control cases as a whole; and 4) in those RF-positive and strongly anti-CCP-positive bronchiectasis patients, 50\% developed RA over a 12-month period. We suggest that screening for RF in bronchiectasis patients and anti-CCP testing in those RF-positive patients will identify a very high risk cohort for subsequent RA development.

0 @ERSpublications

This study suggests lung inflammation/infection plays a role in autoimmunity relevant to rheumatoid arthritis http://ow.ly/xyULR

Elizabeth Perry ${ }^{1,2}$, Chris Stenton ${ }^{3}$, Clive Kelly ${ }^{4}$, Paul Eggleton ${ }^{2}$, David Hutchinson ${ }^{5}$ and Anthony De Soyza ${ }^{6,7}$

${ }^{1}$ Dept of Rheumatology, Barnstaple Hospital, Barnstaple, UK. ${ }^{2}$ University of Exeter Medical School, Exeter, UK. ${ }^{3}$ Dept of Medicine, Royal Victoria Infirmary, Newcastle, UK. ${ }^{4}$ Dept of Rheumatology, Queen Elizabeth Hospital, Gateshead, UK. ${ }^{5}$ Dept of Rheumatology, Royal Cornwall Hospital, Truro, UK. ${ }^{6}$ Lung Immunobiology and Transplantation Group, Institute of Cellular Medicine, Newcastle University, Newcastle, UK. ${ }^{7}$ Sir William Leech Centre, Freeman Hospital, Newcastle, UK.

Correspondence: Elizabeth Perry, North Devon NHS Trust, Rheumatology, Raleigh Park, Barnstaple, EX31 4JB, UK. E-mail: e.perry@nhs.net

Received: April 042014 | Accepted after revision: May 252014 | First published online: June 252014

Support statement: We acknowledge funding support from Arthritis Research UK to E. Perry (grant 19894) and a Higher Education Funding Council for England Senior Lectureship to A. De Soyza. We also acknowledge National Institute for Health Research CLRN support to the recruiting centres via the UK Comprehensive Research Network portfolio.

Conflict of interest: Disclosures can be found alongside the online version of this article at www.erj.ersjournals.com

Acknowledgements: We thank Dr Anne Marie Quirke and Prof. Patrick Venables of the Kennedy Institute, Oxford, UK, for measurement of anti-CCP. We also thank Dr Gill Baker and colleagues of the NIHR Clinical Research Facility, Exeter University Medical School, Exeter, UK, for providing ethically approved healthy control serum samples.

\section{References}

Perry E, Kelly C, Eggleton P, et al. The lung in ACPA-positive rheumatoid arthritis: an initiating site of injury? Rheumatology (Oxford) 2014 [In press DOI: 10.1093/rheumatology/keu195].

Despaux J, Toussirot E, Wendling D. Bronchiectasis and rheumatoid arthritis: frequency and etiopathogenic factors. A literature review. Rev Med Interne 1997; 18: 144-152.

Nielsen SF, Bojesen SE, Schnohr P, et al. Elevated rheumatoid factor and long term risk of rheumatoid arthritis: a prospective cohort study. BMJ 2012; 345: e5244. 
4 Berglin E, Padyukov L, Sundin U, et al. A combination of autoantibodies to cyclic citrullinated peptide (CCP) and HLA-DRB1 locus antigens is strongly associated with future onset of rheumatoid arthritis. Arthritis Res Ther 2004; 6: R303-R308.

5 Hilton AM, Doyle L. Immunological abnormalities in bronchiectasis with chronic bronchial suppuration. Br J Dis Chest 1978; 72: 207-216.

6 Horan MA, Leahy BC, Fox RA, et al. Immunological abnormalities in patients with chronic bronchial suppuration: a possible relationship with endotoxaemia. $\mathrm{Br}$ J Dis Chest 1984; 78: 66-74.

7 Aletaha D, Neogi T, Silman AJ, et al. 2010 rheumatoid arthritis classification criteria: an American College of Rheumatology/European League Against Rheumatism collaborative initiative. Ann Rheum Dis 2010; 69: 1580-1588.

8 Tuomi T, Heliovaara M, Palosuo T, et al. Smoking, lung function, and rheumatoid factors. Ann Rheum Dis 1990; 49: 753-756.

9 Yang DH, Tu CC, Wang SC, et al. Circulating anti-cyclic citrullinated peptide antibody in patients with rheumatoid arthritis and chronic obstructive pulmonary disease. Rheumatol Int 2014; 34: 971-977.

10 Hoffman IE, Peene I, Cebecauer L, et al. Presence of rheumatoid factor and antibodies to citrullinated peptides in systemic lupus erythematosus. Ann Rheum Dis 2005; 64: 330-332.

11 King PT. The pathophysiology of bronchiectasis. Int J Chron Obstruct Pulmon Dis 2009; 4: 411-419.

12 Elliot JG, Jensen CM, Mutavdzic S, et al. Aggregations of lymphoid cells in the airways of nonsmokers, smokers, and subjects with asthma. Am J Respir Crit Care Med 2004; 169: 712-718.

13 Rangel-Moreno J, Hartson L, Navarro C, et al. Inducible bronchus-associated lymphoid tissue (iBALT) in patients with pulmonary complications of rheumatoid arthritis. J Clin Invest 2006; 116: 3183-3194.

14 Nielen MM, van Schaardenburg D, Reesink HW, et al. Specific autoantibodies precede the symptoms of rheumatoid arthritis: a study of serial measurements in blood donors. Arthritis Rheum 2004; 50: $380-386$.

15 Chorus AMJ. Reuma in Nederland: de cijfers. Actualisering 2000. [Rheumatism in the Netherlands: the figures. 2000 update.] Leiden, TNO, 2001.

\section{Human-to-human transmission of Mycobacterium kansasii or victims of a shared source?}

\section{To the Editor:}

Nontuberculous mycobacteria (NTM) are ubiquitous in the environment [1] and can cause opportunistic infections in humans with either an immunological deficit or structurally abnormal lungs, such as in cystic fibrosis, bronchiectasis or chronic obstructive pulmonary disease (COPD) [2]. Unlike Mycobacterium tuberculosis, human-to-human transmission of NTM is generally thought to be uncommon [2-4], although there is evidence that transmission of certain NTM is possible in certain circumstances [5]. Domestic plumbing has also been suggested as a potential source of infection [6].

We describe what we believe to be the best evidence to date of human-to-human transmission of Mycobacterium kansasii in a husband and wife living in East London, UK, an area with a high incidence of tuberculosis ( $\sim 112$ cases per 100000 per year) [7]. A recent survey suggested a UK isolation rate for NTM of around three cases per 100000 per year with no data available for true disease incidence [8].

The first of the couple to be seen in chest clinic was a 69-year-old Caucasian lady who reported a 6-month history of weight loss, reduced appetite and a cough productive of $30 \mathrm{~mL}$ sputum twice daily. Her past medical history was of an episode of severe pneumonia aged 6 years and COPD, having smoked 10 cigarettes per day until 2 years previously. All routine blood tests were unremarkable, including a normal erythrocyte sedimentation rate (ESR) of $11 \mathrm{~mm} \cdot \mathrm{h}^{-1}$ (normal range $10-15 \mathrm{~mm} \cdot \mathrm{h}^{-1}$ ). Chest computed tomography (CT) was ordered, and demonstrated emphysema, right lower lobe bronchiectasis and a calcified granuloma. Three sputum samples were smear negative, but two of her three sputum samples proved culture positive for mycobacteria and she was commenced on standard antituberculous chemotherapy. After the mycobacteria in both samples had been identified as M. kansasii, isoniazid and pyrazinamide were discontinued. She continued on rifampicin and ethambutol for a total of 12 months, and converted to negative cultures after 2 months of treatment. It should be noted that this regimen is not that advocated by the American Thoracic Society [2] but was administered at the discretion of her treating physician.

Within a month of this woman being diagnosed with M. kansasii disease, her husband was referred to the same chest clinic by their general practitioner (GP). This 75-year-old Caucasian male presented with a history of $20 \mathrm{~kg}$ weight loss over 3 years, on a background of 60 pack-years of smoking. He was known to 